\title{
Denoising Algorithm in Fresnel Transform Domain for Electronic Holographic Video System
}

\author{
Hyun-Jun Choi \\ Dept. of Electronic Eng., Mokpo National Maritime University \\ Haeyangdaehak-ro 91, Mokpo-si, Jeonnam, 530-729, South Korea \\ hjchoi@mmu.ac.kr
}

\begin{abstract}
Holography is the optimal and the final choice among seteral techntaves for imaging and displaying the three-dimensional (3D) objects. In this paper, we propose a new denoising algorithm for digital hologram using Fresnel transform (FT). The proposed algorithm initially transforms a digital hologram into frequency-domain data through a FT, and separates the object from the background in the transformed inage. Experimental results show that the technique to reduce noise in a natural 2D imagesuch as a smoothing filter can reduce the noise to improve the image quality for a digital hologram. We expect that the conclusions of this paper will be a very useful technique for further work in the area of signal processing scheme for electronic holographic vided service.
\end{abstract}

Keywords: Digital hologram, Denoising, Noise reduction, Holographic video, Computergenerated hologram

\section{Introduction}

The surge of recent interest into 3D images has led to active researches about digital holography, which is the ultinate goal of 3D imaging in various fields: capturing or producing a digital hologram reconstructing a digital hologram, studying optical materials and devices, and signal processing. Especially, the last one has recently attracted attention. A hologram used to be dealt with from the optical side only. Holographic digitalization, however, has led to various studies on encoding, transmission, storing, encryption, and noise reduction [1,2].

Holograms have been recognized by most image researchers as the final goal of perfect 3D image reconstruetion, because they are exactly the same image, as the original object in free space. Thas, many researchers have working on this, since their invention by Gabor in 1948.

Electronic holograms have been researched since the 1960s. Computational holography is one of these forms of electronic holograms, in which the interference pattern (fringe pattern or digital hologram) is calculated numerically to acquire a hologram. The hologram is uploaded to a spatial light modulator (SLM) and the reference light is exposed to reconstruct the image [3, 4]. The numerically calculated hologram is termed a computer-generated hologram (CGH). The inputs for a $\mathrm{CGH}$, the depth information and the light intensity of each object point, are in digital forms. In addition, the output, the resulting hologram, consists of pixels.

The technique to remove noise from a natural image has been experimented a long time ago, and, subsequently, many noise-removing filters showing superior performances have been published. However, these filters are not suitable to a digital hologram because the noise is at a similar frequency region to the original image data. Some studies $[5,6]$ proposed the 
noise-removing techniques for the digital hologram, using the image processing technique. In [7] proposed a method using a wavelet filter. These techniques expanded the applicability of the methods to the 2-dimensional (2D) images of the digital hologram.

In this paper, a new approach to remove or reduce the noise in the digital hologram is proposed, which uses a frequency transform to increase the correlation between the pixels. A Fresnel Transform is used as the frequency-transform tool. The distribution of the frequencytransformed coefficients resembles one of the reconstructed images [8]. Consequently, the object and the background in the transformed image are separated and analyzed to give the treatment for different noise-removing techniques.

In Chapter 2, the theoretical background and Fresnel transform is described. Chapter 3 explains the denoising method proposed in this paper with a brief review of some previous ones. The experimental results are provided with a following discussion in chapter 4 , and chapter 5 concludes the paper.

\section{Background Theory}

\subsection{Digital Hologram}

In general, a digital hologram is acquired by capturing the phase of interference pattern between the reference light and the object light reflected from an object with CCD camera, and recorded in digital format, as shown in Figure 1. In the case of acquiring a digital hologram optically as Figure 1a, a laser is exposed to condensing lens to produce a parallel light. This light is divided into two lights (the reference wave and the object wave) by a beam splitter. The object wave is illuminated to the object and the reference wave is directly exposed to the CCD camera. These two waves interference each other and the resulting interference pattern is recorded in the CCD camera. To restore the object, the interference pattern is loaded to a SLM and the same light as the reference wave in recording is exposed to the SLM. Then, the primary diffraction ray is generated and the 3D object is reproduced [9].

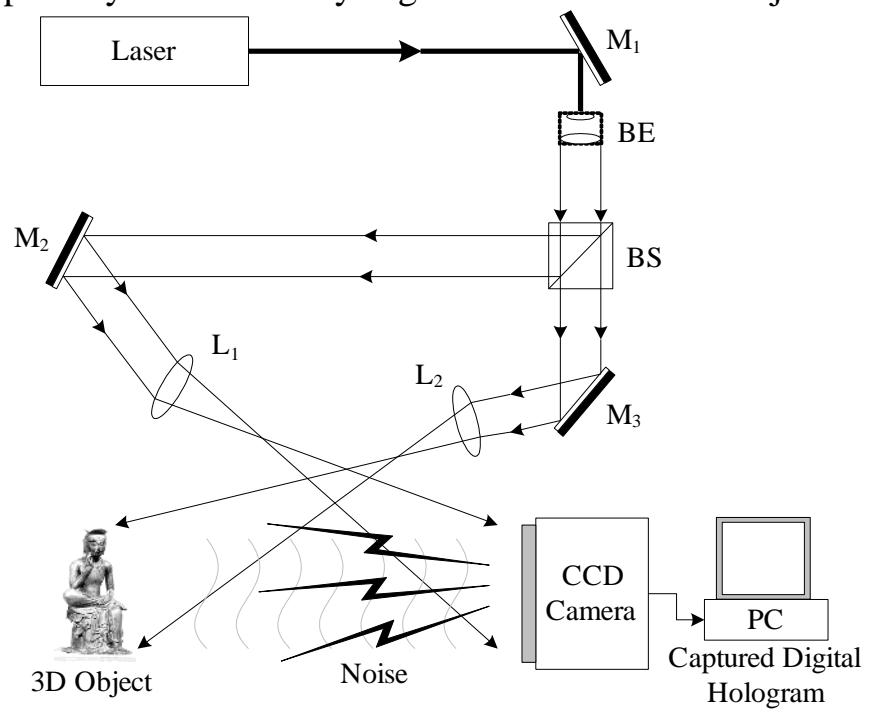

Figure 1. Acquisition Scheme of a Digital Hologram

The use of CCD camera in the acquisition process of a digital hologram inherently includes some noises because of the environments of camera, which dramatically decreases the quality of reconstructed images with the digital hologram. 


\subsection{Fresnel Transform}

The Fresnel diffraction equation for near-field diffraction, is an approximation of Kirchhoff-Fresnel diffraction that can be applied to the propagation of waves in the near field. It is used to calculate the diffraction pattern created by waves passing through an aperture or around an object, when viewed from relatively close to the object. In contrast the diffraction pattern in the far field region is given by the Fraunhofer diffraction equation. Figure 2 show a diffraction geometry for Fresnel diffraction. Here, $E(\xi, \eta, 0)$ is $2 \mathrm{D}$ pattern of optical field on an image plane and $E(x, y, z)$ is the field at output plane a distance away [10].

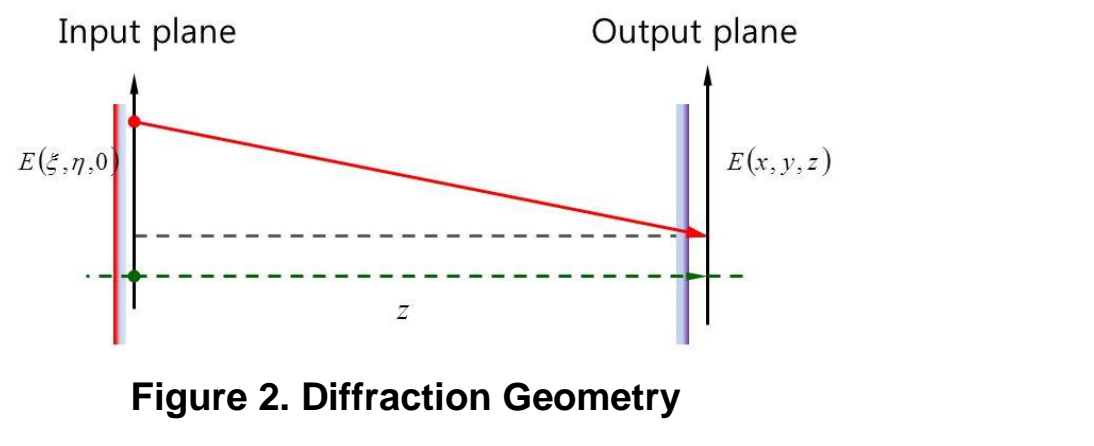

Figure 3 shows an example images, where (a) is a computer graphic image (depth-map in $200 \times 200$ [pixel $^{2}$ ]), (b) is its digital hologram $\left(256 \times 256\right.$ [pixel $\left.\left.^{2}\right]\right)$, and (c) is the result from Fresnel transform of a digitar hologran. The Fresnel transform result is much resembled to the original object (Figutre 3(a)). Qne particular result from this example is that, the value of DC coefficient from a digital hologram does not seem to be so high. But they still have very high values and energies even though they are lower than those a conventional 2D image.
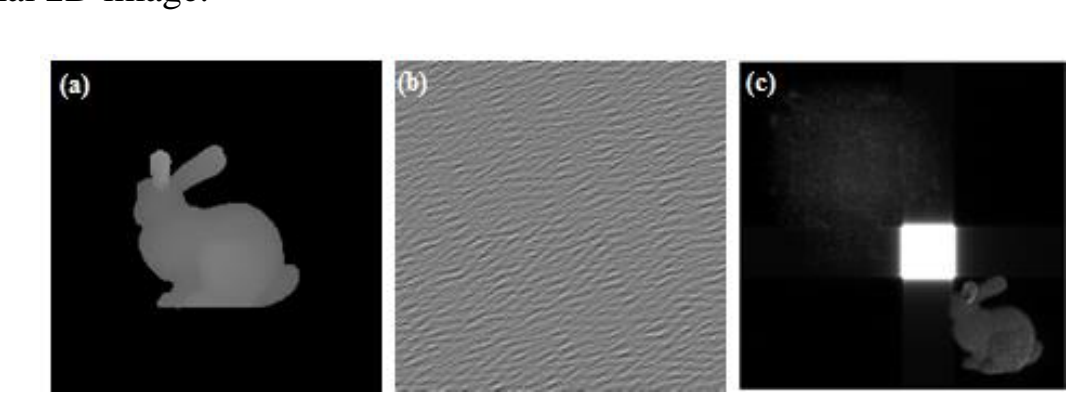

\section{Figure 3. (a) Depth-map of 3D Object, (b) Digital Hologram of (a), (c) Fresnel Transform Result of (b)}

\section{Proposed Algorithm}

As mentioned above, other than the way to create a digital hologram in Figure 2(b) is the Fresnel transform that calculates the interference pattern arithmetically with the brightness map (or color map) and the depth map of the object. This method, as also employed in this paper, is popularly used among experiments. Even this CGH (computer generated hologram) is used, because it is necessary to model the actual acquisition situation as Figure 1 because $\mathrm{CGH}$ does not include the noise introduced during the acquisition process. Converting a 3D object $\mathrm{o}(\mathrm{x}, \mathrm{y})$ to a digital hologram $h(i, j)$ by the CGH technique and adding the noise $\mathrm{n}[0$, $\sigma 2]$ results in the definition of the actual digital hologram $h^{\prime}(i, j)$ as in Eq. (1).

$$
h^{\prime}(i, j)=h(i, j)+n(i, j)
$$


where $n(i, j)$ is the noise at the position of $(i, j)$ on the assumption that the noise is Gaussian noise with the average of 0 and the standard deviation of $\sigma 2$.

As shown in Figure 4(c), the FTed of the digital hologram results in a similar pixel distribution to the original object although the position and the size somewhat change. Using this characteristic, the present paper proposes a new noise reduction algorithm regarding the FTed image as a nature image.

For denoising, Fresnel transform is performed by regarding a digital hologram as a natural 2D image. Figure 4(c) shows a FT result image. As shown in Figure 4(c) and (d), the coefficient distributions of digital hologram after the FT show relatively high correlations of the pixel values compared to the ones before the FT as shown in Figure 4(a). For the distributions in Figure 4(c) and (d), the pixel values inside the objects show relatively high correlations compared to the exterior pixels to the object. Also, the pixel values outside the object are relatively very low to the ones inside the object, which is the sane restit from the actual measurement. That is, the characteristics of the coefficients inside the object are different from the ones of the coefficients outside the object. Therefore, this paper separates the object from the background and proposes a different noise reduction scheme for each of them.

From such characteristics, the following conclusions can be made. Because the local region of a digital hologram includes information for the entire object, we must encrypt the entire hologram rather than the segmented hologram.

The denoising methodology is the following:

(1) Fresnel transform

(2) Selection of denoising region

(3) Denoising using proposed methods

(4) Inverse Fresnel transform

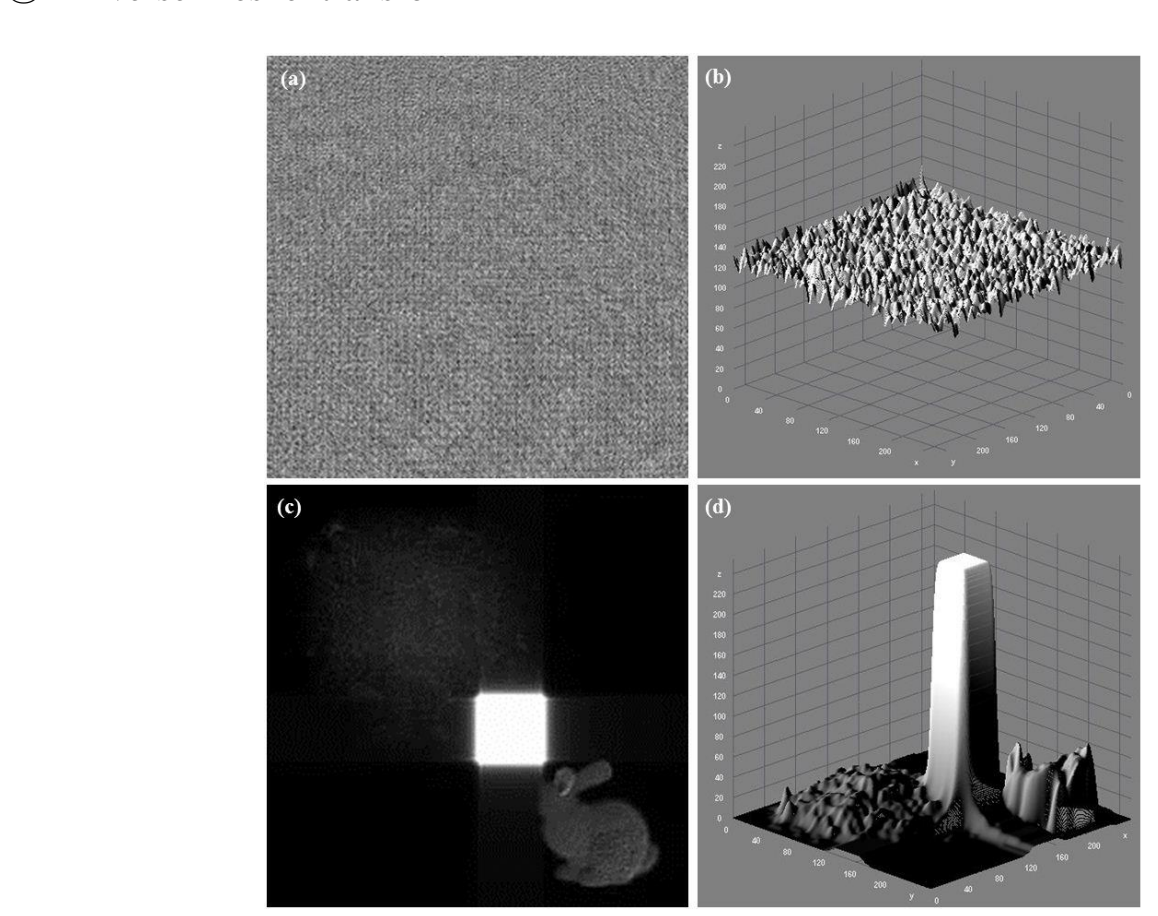

Figure 4. (a) Digital Hologram , (b) Pixel Distribution of (a), (c) Fresnel Transform Result of (a), (d) Pixel Distribution of (c) 
To apply the proposed algorithm, digital holograms of 3D object (depth-map, intensity) are generated using the CGH method. The proposed algorithm is applied to them. The encryption results are verified numerically using peak noise-to-signal ratio (PSNR) and normalized correlation (NC). In addition to the numerical statistics, visual observation is used to determine the encryption efficiency. The size of a digital hologram is $1,024 \times 1,024\left[\right.$ pixel $\left.^{2}\right]$.

We divide the two regions in FT domain, whose scheme is shown in Figure 5. This paper separates the object and the background in the FT domain by using the distribution characteristics of those coefficients, and proposes each different noise reduction technique.

Background region is to remove/reduce noise outside the object for the FTed image. As explained above, the coefficients outside the object have relatively very low correlations with the ones inside the object. Thus, removing the values outside the object induces a very low influence on the quality of the image. So, Background is substituted with ' 0 '

In object region, the distribution characteristics in the statistical histogram of the FTed coefficients are used. To obtain the distribution characteristics, more than 100 digital holograms produced by the calculation $(\mathrm{CGH})$ were tested. The denoising using statistical histograms is as follows.

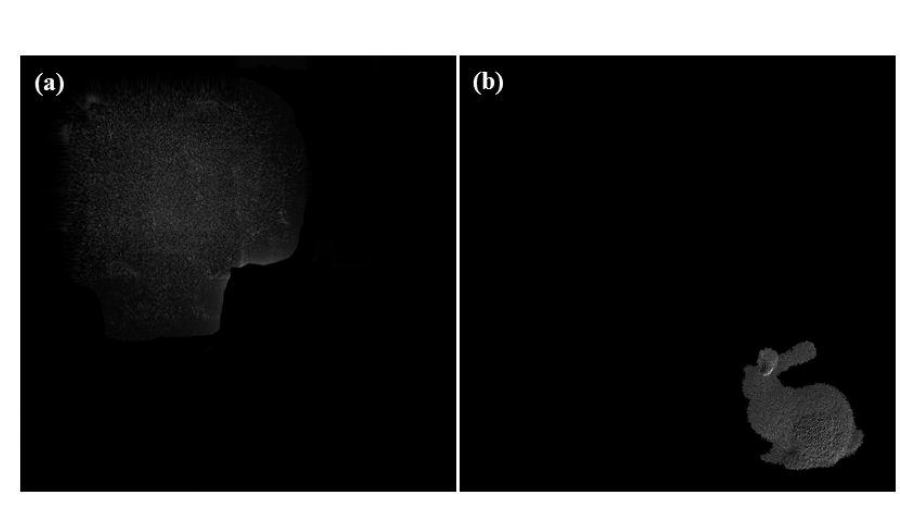

\section{Figure 5. Two Regjons for Denoising in Fresnel Domain}

The object and the background are separated and a Butterworth lowpass filter [11] is applied to the object. A Butterworth lowpass filter is a kind of low-pass filter which performs a weighted average for the target pixel and the surrounding ones within the filtering window. A Butterworth lowpass filter can be described as Eq. (2),

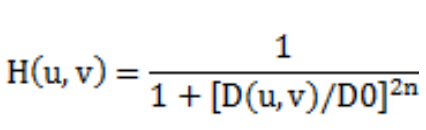

where $D O$ is a positive constant and $D(u, v)$ is the distance between a point $(u, v)$ in the frequency domain and the center of the frequency rectangle.

\section{Experimental Results}

The proposed in the previous section have been experimented with more than 100 digital holograms (CGHs). To make a digital hologram noisy, we added Gaussian noise as shown in Eq. (1). For the objective measurement of the visual quality of a reconstructed image, the PSNR (peak signal to noise ratio) as shown in Eq. (3) has been measured, while the subjective comparison of the image quality has been accomplished with the reconstructed images on an optical system as shown in Figure 1. 


$$
\operatorname{PSNR}(d B)=10 \log _{10} \frac{255^{2}}{\frac{1}{X Y} \sum_{x, y}\left(I_{x, y}-I_{x, y}^{\prime}\right)^{2}}
$$

Here, $I_{x, y}$ and $I_{x, y}^{\prime}$ are the values in the position of $(\mathrm{x}, \mathrm{y})$ of the noise-free and the noisy reconstructed image, respectively. $X$ and $Y$ are the numbers of pixels in the horizontal and vertical direction in the reconstructed image, respectively.

A smoothing filter which used to reduce noise in natural images was applied to reduce the noise of digital holograms. That is, the filter has been applied to the data which occurred due to FTed with a noisy digital hologram. Figure 6 shows an example of the experimental results.

As can be imagined, the experimental results showed that the image qualities of the reconstructed images after filtering were worse than the one without filtering. The PSNR values in Figure 6(c) and Figure 6(d) (without filtering) were 16.33 [dBl and 17.81[dB], respectively. But by applying our methods, the image qualities were imaproved by at least $3.2[\mathrm{~dB}]$ in the PSNR average.
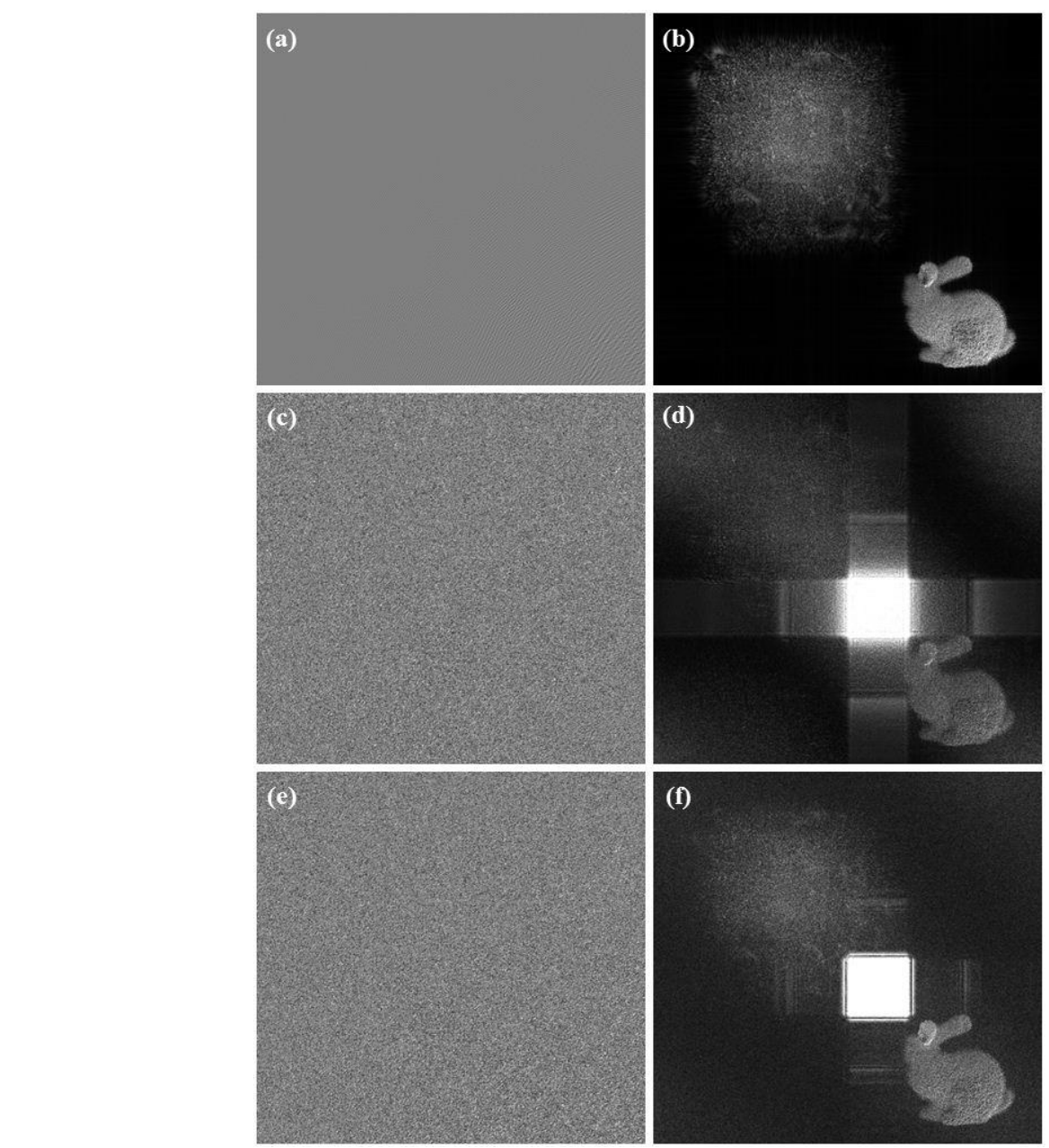

Figure 6. Experimental Results (a) Digital Hologram , (b) Reconstruction Image of (a), (c) Noisy of (a), (d) Reconstruction Image of (c), (e) Denoising by Proposed Method, (f) Reconstruction Image of (e) 


\section{Conclusion}

This paper proposed an algorithm to reduce noise added during the acquisition and transmission process of a digital hologram and cause dramatic degradation in the image quality. This algorithm is used to separate the image part from the background data. The Fresnel transform frequency domain data were treated as natural images by separating the object from the background by contouring and proposing of denoising methods.

Experimental results showed that the technique to reduce noise in a natural 2D image such as a smoothing filter could not reduce the noise to improve the image quality for a digital hologram. But by applying our methods, the image qualities was improved by at least 3.2 $[\mathrm{dB}]$ in the PSNR average.

Consequently, we could be sure that the proposed denoising schemes can provide various digital holographic videos for various environments. Thus, we are expecting that the proposed schemes can be used as a good basis for further research on denoising, as well as for real applications such as a holographic TV. As for the interpolation method, it will be one of our future research topics to find an optimal method to increase the object quality.

\section{Acknowledgment}

This work was supported by the National Research Foundation of Korea Grant funded by the Korean Government (MEST) (NRF-2010-0022080).

\section{References}

[1] O. Matoba, T. J. Naughton, Y. Frauel, N. Bertaux and B. Javidi, "Three-dimensional object reconstruction using phase-only information from a digital hologram", Proc, SPIE 4864, (2002).

[2] Y. Piao, Y. M. Kwon, M. Zhang and J. J. Lee, Accelerated Generation Algorithm for an Elemental Image Array Using Depth Information in Computational Integral Imaging", Journal of information and communication convergence engineering, vol. 11, no. 2, (2013), pp. 132-138.

[3] A. E. Shortt, T. J. Naughton and B. Javidi, "A companding approach for nonuniform quantization of digital holograms of three-dimensional objects", Optics Express, vol. 14, no. 12, (2006), pp. 5129-5134.

[4] X. Cai and H Wang, "Study of relationship between recording wavelength and hologram compression", Optics Communications, vor 265, ho. 1, (2006), pp. 111-115.

[5] J. G. Sucerquia, J. A. H. Ramirez and D. V. Prieto, « Reduction of speckle noise in digital holography by using digital image processing?, Optik optics, vol. 116, (2005), pp. 44-48.

[6] X. Cai and H. Wang The influence of hologram aperture on speckle noise in the reconstructed image of digital holography and its reduction", Optics Communications, vol. 281, no. 2, (2008), pp. 232-237.

[7] L. C. Lin, "An (error detection and recovery algorithm for the transmission of digital holography over noisy channels", Optics Communications, vol. 281, no. 5, (2008), pp. 1008-1016.

[8] A. Sharma, G. Sheoran, Z. A. Jaffery and Moinuddin, "Improvement of signal-to-noise ratio in digital holography using wavelet transform", Optics and Lasers in Engineering, vol. 46, no. 1, (2008), pp. 42-47.

[9] 14. J Cho1, "Fast Generation Algorithm of Digital Hologram based Depth Difference Temporal Filtering", International Journal of Multimedia and Ubiquitous Engineering, vol. 8, no. 6, (2013), pp. 171-180.

[10] http://en.wikipedia.org/wiki/Fresnel_diffraction

[11] R. C. Gonzalez and R. E. Woods, «Digital Image Processing », $3^{\text {rd }}$ Edition, Pearson Prentice Hall, (2009), pp. 291-298. 


\section{Author}

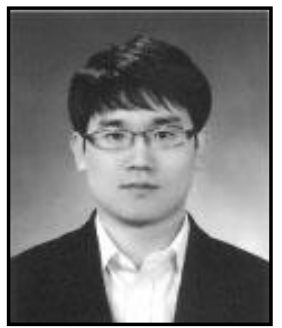

Hyun-Jun Choi, he received his M.S. and Ph.D. degrees in 2005 and 2009 from Dept. of Electronic Materials Engineering of Kwangwoon University in Seoul, Korea. He is currently an assistant professor with the department of Electronic Engineering, Mokpo National Maritime University. He is research interests are in optical image processing and 3D display.

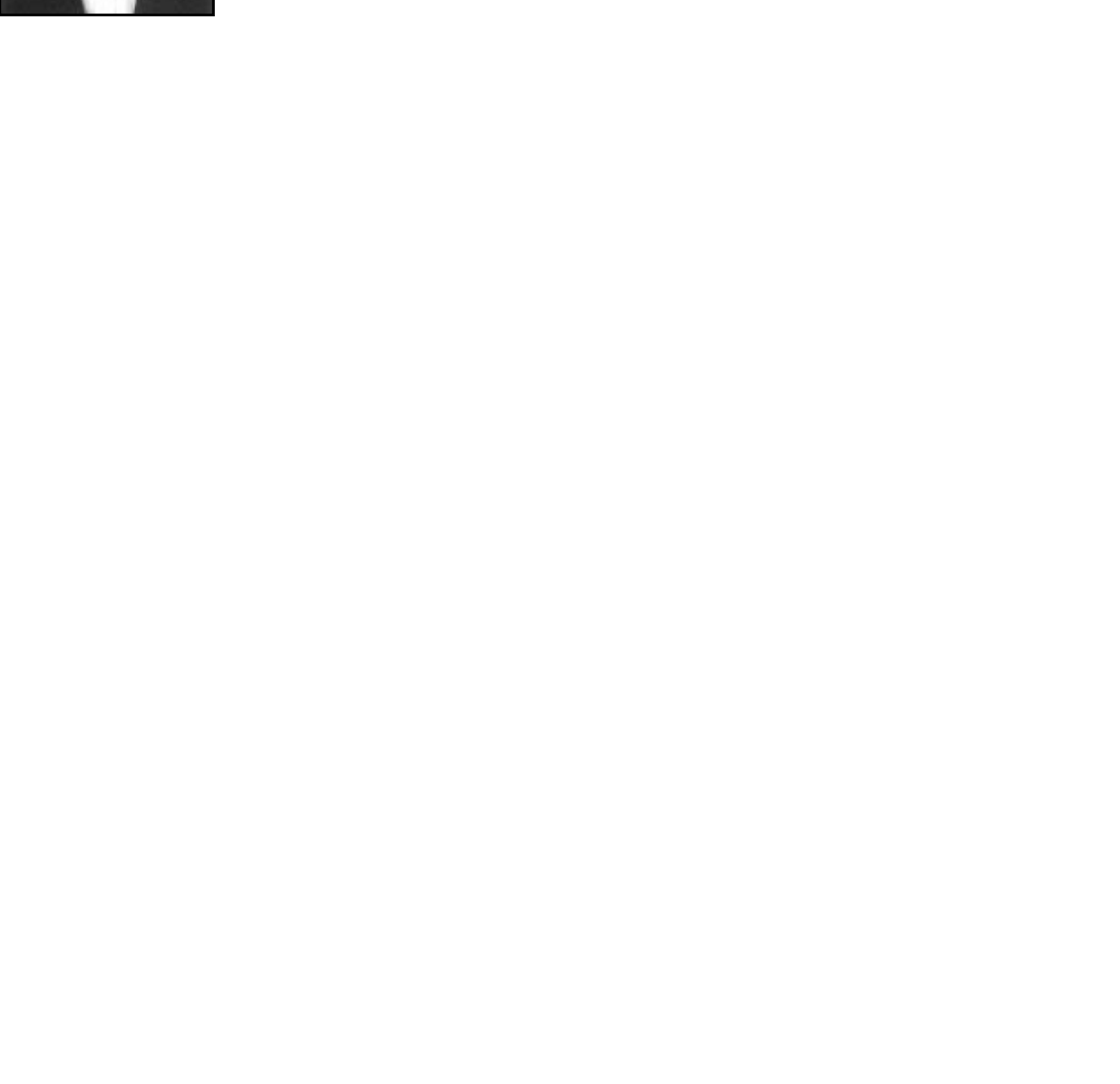

